\title{
Patient characteristics affect the response to ketamine and opioids during the treatment of vaso-occlusive episode-related pain in sickle cell disease
}

\author{
Raissa Nobrega ${ }^{1}$, Kathy A. Sheehy ${ }^{1}$, Caroline Lippold ${ }^{1}$, Amy L. Rice ${ }^{1}$, Julia C. Finkel ${ }^{1}$ and Zenaide M.N. Quezado ${ }^{1,2}$
}

BACKGROUND: N-methyl-D-aspartate receptor activation has been implicated in the pathobiology of inflammatory, nociceptive and neuropathic pain, opioid tolerance, opioidinduced hyperalgesia, and central sensitization. Some of those mechanisms underlie sickle cell disease(SCD)-associated pain. METHODS: We conducted an exploratory cohort study of SCD patients who during vaso-occlusive episodes (VOEs) received subanesthetic doses of the $\mathrm{N}$-methyl-D-aspartate receptor antagonist, ketamine, as an adjunct to opioids. We sought to identify predictors of changes in pain scores and of the percentage of ketamine infusions associated with meaningful changes ( $\geq 20 \%$ reduction) in pain and opioid consumption.

RESULTS: Eight-five patients received 181 ketamine infusions for VOE-associated pain. Combined with opioids, ketamine yielded significant decrease in pain scores and opioid consumption. Ketamine administered to males and to younger patients yielded greater pain score decrease compared with females $(P=0.013)$ and older patients $(P=0.018)$. Fifty-four percent of infusions yielded meaningful reductions in pain scores, and in multivariate analysis, sex, age group, pain location, and infusion duration independently predicted pain score changes.

CONCLUSION: This study suggests that in SCD patients admitted with VOE-associated pain, ketamine has age- and sex-dependent effects. These data can inform sample and effect size calculations for controlled trials to determine which SCD patients would benefit most from ketamine.

A mong patients with sickle cell disease (SCD), pain resulting from vaso-occlusive episodes (VOEs) continues to be the principal reason for hospital admission (1-3). Unfortunately, the management of VOEs has changed little since the initial studies of SCD-associated pain $(1,4,5)$ and still consists of opioids, non-steroidal anti-inflammatory drugs, and intravenous hydration. Although opioids continue to be the mainstay of analgesic therapy during VOEs, some patients have continued and increasing pain despite receiving escalating doses of opioids (6). This phenomenon, in some patients, is believed to result from opioid-induced hyperalgesia or tolerance or from the fact that VOE-related pain could be partially unresponsive to opioid modulation (3). In addition, an increasing body of literature indicates that, as SCD patients reach adolescence and adulthood, a significant number will develop ongoing and chronic pain, which appear to have a neuropathic component (7-10). In turn, the presence of a pre-existing neuropathic process adds complexity to the treatment of acute VOE-associated pain as opioids are often ineffective in neuropathic pain. Although there are a number of ongoing clinical trials of therapies targeting potential mechanisms of VOEs in SCD (5), therapies that effectively treat VOE-associated SCD pain in patients chronically exposed to opioids are currently unavailable.

Ketamine, a non-competitive $\mathrm{N}$-methyl-D-aspartic acid (NMDA) receptor antagonist, has been used as a general anesthetic since the 1960s. More recently, ketamine in subanesthetic doses has been explored as an adjuvant to opioids to treat chronic and acute pain and opioid-induced hyperalgesia or tolerance in children and adults (11-14). The rationale supporting the use of subanesthetic ketamine for pain management includes preclinical evidence implicating activation of NMDA receptors in the pathobiology of central sensitization and inflammatory, nociceptive, and neuropathic pain (15).In addition, in animals and humans, the activation of NMDA receptors has been shown to underlie the development of opioid tolerance and opioid-induced hyperalgesia (16). There is also some clinical evidence suggesting that, when given in conjunction with opioids in the postoperative period, ketamine ameliorates pain and reduces opioid requirements in opioid-naive patients (14). Further, intraoperative administration of ketamine to patients with chronic back pain and opioid dependence decreases opioid requirements and reduces the incidence of late postoperative pain after spinal fusion (17). Therefore, given the reported analgesic and opioid-sparing effects in acute and chronic pain as well as a favorable safety profile, researchers have argued

\footnotetext{
'Division of Anesthesiology, Pain, and Perioperative Medicine, The Sheikh Zayed Institute for Pediatric Surgical Innovation, Children's Research Institute, Children's National Health System, George Washington University School of Medicine and Health Sciences, Washington, DC; ${ }^{2}$ Pediatric Anesthesia and Critical Care, Department of Perioperative Medicine, National Institutes of Health Clinical Center, National Institutes of Health, Bethesda, Maryland. Correspondence: Zenaide M.N. Quezado (zquezado@nih.gov) 
that ketamine might have a role in modulating SCD pain $(18,19)$.

Several groups have reported the use of intravenous $(18,20$ 22) and intranasal ketamine (23) in SCD patients presenting with VOEs. Here, we conducted an exploratory study on a large cohort of SCD patients who during admissions for VOEs were treated with subanesthetic doses of ketamine. We sought to determine the feasibility of this therapeutic modality in SCD patients during hospital admission for VOE, and to test the hypotheses that patient and infusion characteristics could affect and predict the changes in pain scores and opioid consumption associated with ketamine administered in combination with opioids.

\section{METHODS}

\section{Data Collection}

The Children's National Health System Institutional Review Board approved the study and waived the need for informed consent as the collected data were de-identified and the study posed minimal risk. We evaluated the subset of inpatients with SCD who, between January 2006 and April 2014, were admitted for VOEs and received subanesthetic doses of ketamine for pain therapy. Patients whose ketamine infusion was not ordered by the pain medicine team, who lacked complete records of pain scores and/or opioid consumption during the study period (see times below), and who had acute chest syndrome were excluded. From the electronic medical record we collected patients' sex, age, race, pain location, genotype, pain scores, and daily opioid consumption. We considered patients with pain in three or more locations to have generalized pain. In addition, we sought to record any and all documentations of hemodynamic changes, hallucinations, or dysphoria and of any therapy administered to treat any of those possible known side effects of ketamine. Considering all recorded pain scores using the Wong-Baker FACES, Face, Legs, Activity, Cry, and Consolability (FLACC), or Numeric Rating Scale (NRS), as appropriate, we determined the average daily pain scores before ( $24 \mathrm{~h}$ prior) and after $(24 \mathrm{~h}, 48 \mathrm{~h}$, and day after ketamine discontinuation) ketamine infusions were initiated. We used the opioid conversion tool available at website www.globalrph. com/narcoticonv.htm (24) to determine daily opioid consumption at times indicated above (oral morphine equivalents/per body weight in $\mathrm{kg}$ ).

\section{Outcomes}

The primary outcome was overall change in pain scores from the baseline measurement to the day after ketamine infusion. Secondary outcomes included overall average change in opioid consumption (oral morphine equivalent) and proportion of ketamine infusions associated with a clinically meaningful change in pain scores and opioid consumption. In this investigation, we defined clinically meaningful change as a $20 \%$ or greater reduction in pain scores or in morphine-equivalent consumption from baseline to the day after ketamine discontinuation (day after ketamine discontinuation-first day of ketamine/first day $\times 100$ ). Although $20 \%$ is an arbitrary value, it is larger than the value (a $12.5 \%$ decline) found in previous studies to reflect a clinically meaningful change in pain scores in adolescents with chronic pain (25) or in SCD patients being treated for pain (26).

\section{Ketamine Administration}

In our institution, we have used subanesthetic ketamine (defined as $\leq 1 \mathrm{mg} / \mathrm{kg} / \mathrm{h}$ ) for the management of acute and chronic pain in children and adolescents. Recently, we reported the use of ketamine in outpatient (12) and inpatient (27) settings to treat acute and chronic pain in children, adolescents, and young adults whose response to opioids is suboptimal or its use is associated with opioid tolerance or opioid-induced hyperalgesia $(27,28)$. The pain-medicine team identifies patients who might benefit from subanesthetic ketamine and orders its administration. The indications for subanesthetic ketamine include inadequate analgesia with standard pain therapy (patient-controlled analgesia), evidence of opioid tolerance, and associated undesirable side effects with escalating opioid doses, or opioid-induced hyperalgesia. Contraindications for ketamine include hypersensitivity to ketamine, history of psychosis, intracranial hypertension, labile hypertension, cardiac arrhythmias, and age less than 3 months. During all ketamine infusions, patients were kept on pulse oximeter, heart and respiratory rate, and blood pressure monitoring continuously. As suggested by the order set, ketamine infusions were typically started at $0.05-0.4 \mathrm{mg} / \mathrm{kg} / \mathrm{h}$ and could, in some cases, be titrated up to $1 \mathrm{mg} / \mathrm{kg} / \mathrm{h}$ according to pain intensity as directed by the pain medicine team.

\section{Statistical Analysis}

In this study, we examined two analytical cohorts because many of the patients were admitted to the hospital more than once during the study period and received ketamine. In order to explore whether repeated ketamine treatment was associated with differences in the study outcomes, we examined two analytical cohorts. The first cohort included data from the initial ketamine infusion of each SCD patient (if complete outcome data were available), whereas the second cohort (the all-admissions cohort) included data from all ketamine infusions received by all SCD patients during any hospital admission (with complete outcome data) during the entire study period.

The distributions of pain scores, daily opioid consumption, and changes over time in raw pain scores and daily opioid consumption were tested for normality in both analytical cohorts. Because the distributions were somewhat skewed, both a paired $t$-test as well as a non-parametric equivalent (the Wilcoxon sign-rank test) were used to test for statistical significance within individual changes between the baseline and post-treatment measurements.

Boxplots were used to examine how the individual-level changes in raw pain scores and daily opioid consumption were distributed across the subgroups of six patient characteristics (genotype, age category, gender, infusion duration, pain location, and the sequence of ketamine treatment (first vs. all hospital admissions)) and the distributions were tested for normality. Because the data were skewed for certain characteristics, non-parametric tests were used for the analysis of both study cohorts in order to maintain a consistent approach.

We used the Kruskal-Wallis test to compare the distribution of values across the subgroups of each patient characteristic separately. When an overall difference was detected, Dunn's test (with a Bonferroni adjustment for multiple comparisons) was used to compare all possible pairs of subgroups and identify any statistically significant detectable differences.

The proportion of patients (95\% confidence interval) who achieved a clinically meaningful (20\% or greater) reduction in pain scores and daily opioid consumption were graphed in order to visually compare how the proportions varied across the subgroups of each patient characteristic separately. Fisher's exact test was used to examine whether any of the variations were statistically significant and a non-parametric test for trend was used to examine whether the proportions systematically increased or decreased across the ordered age groups or by the duration of ketamine infusion.

Multivariate mixed effects linear and logistic regression analyses were used to identify which, if any, of those factors still predicted statistically significant differences after taking all of the other patient characteristics into account. The mixed effects model applied to the all-admissions cohort included patient as a random effect in order to account for the fact that individual patients contributed data from multiple hospital admission. Post hoc tests were then used to identify statistically significant differences within the subgroups of individual patient characteristics.

Stata 14.1/IC (College Station, TX, USA) was used to conduct all analyses. $P$ values $<0.05$ were considered statistically significant except when Bonferroni's correction for multiple comparisons was 


\section{Ketamine for pain management during VOE $\quad$ Articles}

applied. In those instances, the statistically significant $P$ value was calculated as $0.05 /$ the total number of comparisons.

\section{RESULTS}

\section{Study Cohorts}

We identified 441 ketamine infusions containing a complete record of the outcome measures of interest at times indicated in the methods (27). Of those, 181 were administered to patients admitted with VOEs. We then examined two cohorts (first ketamine administration and all ketamine administrations (the all-admissions cohort)) in order to explore whether the sequence of ketamine treatment was associated with differences in the study outcomes. The first admission cohort included data from the first hospital admission when ketamine was administered to 80 different patients. The all-admissions cohort contained data from 181 ketamine infusions delivered to 85 different patients (five patients with multiple admissions had incomplete documentation of the outcome measurements during their first hospital admission) each of whom was admitted to the hospital between 1 and 11 times during the study period.

Table 1 lists patient demographics both at the time of their first infusion and including all ketamine infusions. Considering the all-admissions cohort, $14 \%$ of ketamine infusions lasted 2 days, 29\% 3, 21\% 4, 14\% 5, and 22\% 6 or more days. Table 2 lists pain locations recorded in the study cohorts. Among all patients included in the study, 48 of the 85 patients (56\%) were admitted with VOEs and treated with ketamine only once, 24 (28\%) received ketamine during two to four admissions, and the remaining 13 (15\%) received ketamine during five to eleven admissions.

During the admissions included in this study, there were no records of neuropsychotropic or hemodynamic untoward effects nor was there evidence of treatment of these possible side effects during ketamine infusions.

\section{Primary Outcome}

In previous studies, we showed that pain scores measured on the day before and on the first day of ketamine infusions are similar (27). Therefore, pain scores obtained on the first day of infusion were considered the baseline measurement in this analysis. Table 3 lists baseline and post-treatment means and $95 \%$ confidence interval pain scores, and pain score changes on the study cohorts. Overall, during the first and all admissions for VOEs, comparing pain scores on the first infusion day with those on the day after ketamine discontinuation, ketamine infusions in combination with opioids were associated with significant decrease in pain intensity, all $P<0.001$ (Table 3).

Considering all infusions $(N=181)$, the changes in pain scores associated with ketamine (within-patient change, Figure 1) varied according to sex, age, and pain location, but not according to genotype $(P=0.920$, Figure 1d) or infusion duration $(P=0.238$, data not shown). Specifically, males had greater decrease in pain scores compared with females $(P=0.013$, Figure 1a). Ketamine infusions
Table 1. Demographic data from patients at the time of their first ketamine infusion as well as during all ketamine infusions included in this study ${ }^{a}$

\begin{tabular}{|c|c|}
\hline Characteristic & $N(\%)$ \\
\hline \multicolumn{2}{|l|}{ Patients at the time of first infusion (80) } \\
\hline \multicolumn{2}{|l|}{ Sex } \\
\hline Female & $41(51 \%)$ \\
\hline Male & $39(49 \%)$ \\
\hline Age (years), median $\left(25^{\text {th }}-75\right.$ th $)$ & $15(13-17)$ \\
\hline \multicolumn{2}{|l|}{ Race/ethnicity } \\
\hline African American & 79 (99\%) \\
\hline Unknown & $1(1 \%)$ \\
\hline \multicolumn{2}{|l|}{ All ketamine infusions (181) } \\
\hline \multicolumn{2}{|l|}{ Sex } \\
\hline Female & 99 (55\%) \\
\hline Male & $82(45 \%)$ \\
\hline Age (years), median (25th-75th) & $16(13-18)$ \\
\hline \multicolumn{2}{|l|}{ Age group (years) } \\
\hline $2-11$ & $34(19 \%)$ \\
\hline $12-15$ & $49(27 \%)$ \\
\hline $16-18$ & $64(35 \%)$ \\
\hline $19-21$ & $34(19 \%)$ \\
\hline \multicolumn{2}{|l|}{ Genotype } \\
\hline $\mathrm{HbSS}$ & $120(66 \%)$ \\
\hline $\mathrm{HbSC}$ & $40(22 \%)$ \\
\hline \multicolumn{2}{|l|}{ Other } \\
\hline $\mathrm{HbS} / \beta 0$ thalassemia & $19(10 \%)$ \\
\hline $\mathrm{HbS} / \beta+$ thalassemia & $1(1 \%)$ \\
\hline $\mathrm{HbSS} / \mathrm{a}$ thalassemia & $1(1 \%)$ \\
\hline
\end{tabular}

administered at ages 2-11 years had greater and those administered to $12-15$ years lesser decrease in pain scores $(P=0.018$, for overall difference Figure $\mathbf{1 b})$. In addition, greater reductions in pain scores were observed in patients with back and abdominal pain, whereas lesser reductions were observed in patients with chest pain $(P=0.004$, for overall difference, Figure 1c).

\section{Secondary Outcomes}

Overall, during the first and all admissions for VOEs, comparing daily opioid consumption at baseline with the day after discontinuation, ketamine infusions were associated with significant decreases in opioid consumption, all $P<0.001$ (Table 3$)$. In addition, considering all infusions $(N=181)$, the changes in opioid consumption associated with ketamine (within-patient change, Figure 1) varied according to age, but not according to sex $(P=0.180$, Figure 1e), pain location $(P=0.474$, Figure 1g), or infusion duration $(P=0.284$, data 


\section{Articles | Nobrega et al.}

not shown). Infusions administered at ages $2-11$ years yielded greater and those administered at 19-21 years yielded lesser decrease in opioid consumption $(P=0.029$, for overall difference, Figure 1f). Regarding genotype, ketamine infusions administered to patients with hemoglobin SC (HbSC) showed a trend toward lesser reduction in opioid consumption compared with HbSS and all other genotypes $(P=0.068$, for overall difference Figure $\mathbf{1 h})$.

\section{Frequency of Meaningful Reduction in Pain Score and Opioid Consumption}

We then examined the frequency of ketamine infusions that were associated with clinically meaningful ( $\geq 20 \%)$ reductions in pain scores and/or opioid consumption (Figure 2). Overall, $54 \%$ of all infusions yielded clinically meaningful reductions in pain scores. However, the proportion of ketamine infusions associated with clinically meaningful reductions in pain scores varied according to sex, age, and pain location, but not

Table 2. Pain location described by patients at their first and during all admissions for vaso-occlusive episodes when ketamine was administered as an opioid adjuvant ${ }^{\mathrm{a}}$

\begin{tabular}{lc}
\hline First ketamine infusion (80) & \\
Generalized & $30(38 \%)$ \\
Lower extremity & $18(22 \%)$ \\
Chest & $13(16 \%)$ \\
Back & $6(8 \%)$ \\
Upper extremity & $7(9 \%)$ \\
Abdomen & $6(8 \%)$
\end{tabular}

\begin{tabular}{lc} 
All ketamine infusions (181) & \\
Generalized & $60(33 \%)$ \\
Lower extremity & $42(23 \%)$ \\
Chest & $35(19 \%)$ \\
Back & $16(9 \%)$ \\
Upper extremity & $15(8 \%)$ \\
Abdomen & $13(7 \%)$ \\
\hline
\end{tabular}

${ }^{\text {a }}$ ain location was recorded as documented in the pain medicine team consultation. according to genotype $(P=0.635$, Figure $2 \mathrm{~d})$ or infusion duration $(P=0.556$, data not shown). Specifically, a greater proportion of ketamine infusions administered to males was associated with clinically meaningful reductions in pain scores compared with those administered to females $(P=0.034$, Figure 2a). Infusions administered to patients between 2 and 11 years were associated with the highest, whereas those administered to patients between 12 and 15 years with the lowest proportion of a clinically meaningful reduction in pain scores $(P=0.018$, for overall difference Figure $2 \mathbf{b})$. In addition, infusions administered to patients with back, abdominal, and lower extremity pain had the highest, whereas those administered to patients with chest pain had the lowest proportion of a clinically meaningful reduction in pain scores $(P=0.019$, for overall difference Figure $2 \mathrm{c}$ ).

Regarding opioid consumption, $56 \%$ of all ketamine infusions were associated with clinically meaningful $(\geq 20 \%)$ reductions in opioid consumption. However, the proportion of infusions associated with clinically meaningful reduction in opioid consumption varied according to sex, age, genotype, and infusion duration (data not shown), but not with pain location $(P=0.902$, Figure $2 \mathrm{~g})$. A significantly greater proportion of ketamine infusions administered to males was associated with clinically meaningful (20\% or greater) reductions in opioid consumption compared with those administered to females $(P=0.010$, Figure 2e). Infusions administered to patients between 2 and 11 years had the highest, and those to patients between 19 and 21 years had the lowest proportion of infusions associated with clinically meaningful reductions in opioid consumption $(P=0.024$, for overall difference Figure 2f). In addition, the lowest proportion of infusions associated with clinically meaningful reductions in opioid consumption were those administered to patients with HbSC genotype and the highest were those administered to patients with $\mathrm{HbS} / \beta^{0}$ thalassemia, $\mathrm{HbS} / \beta^{+}$ thalassemia, or $\mathrm{HbSS} / \alpha$ thalassemia $(P=0.021$ for overall difference, Figure $2 \mathbf{h}$ ). Lastly, the highest proportion of infusions associated with clinically meaningful reduction in opioid consumption were those administered for 3-6 days and the lowest were those administered for 2 or for 7 or more days $(P=0.038$, for overall difference, data not shown).

Table 3. Pain scores, opioid consumption, and changes over time during the first and all admissions for VOEs when ketamine was administered $^{\mathrm{a}}$

\begin{tabular}{|c|c|c|c|c|}
\hline Outcomes & Baseline mean $(95 \% \mathrm{Cl})$ & After ketamine discontinuation mean $(95 \% \mathrm{Cl})$ & Change mean $(95 \% \mathrm{Cl})$ & $P$ value \\
\hline \multicolumn{5}{|c|}{ First ketamine infusion (80) } \\
\hline Pain scores & $7.49(7.10,7.87)$ & $4.62(3.95,5.29)$ & $-2.87(-3.48,-2.25)$ & $<0.001$ \\
\hline Opioid consumption & $3.36(2.79,3.92)$ & $2.06(1.64,2.47)$ & $-1.30(-1.94,-0.67)$ & $<0.001$ \\
\hline
\end{tabular}

All ketamine infusions (181)

\begin{tabular}{|c|c|c|c|c|}
\hline Pain scores & $7.81(7.58,8.05)$ & $5.44(5.01,5.89)$ & $-2.38(-2.75,-2.00)$ & $<0.001$ \\
\hline Opioid consumption & $3.10(2.75,3.44)$ & $2.22(1.90,2.54)$ & $-0.88(-1.32,-0.44)$ & $<0.001$ \\
\hline
\end{tabular}

$\mathrm{Cl}$, confidence interval; VOE, vaso-occlusive episode

${ }^{a}$ Daily opioid consumption is shown as oral morphine equivalent $(\mathrm{mg} / \mathrm{kg})$ administered over $24 \mathrm{~h}$. 


\section{Predictors of Response to Ketamine Infusion}

We then conducted a multivariate analysis to identify predictors of the response to ketamine as an opioid adjunct in the all-admissions cohort while accounting for the fact that individual patients contributed data from multiple hospital admissions. We found that sex, age group, pain location, and
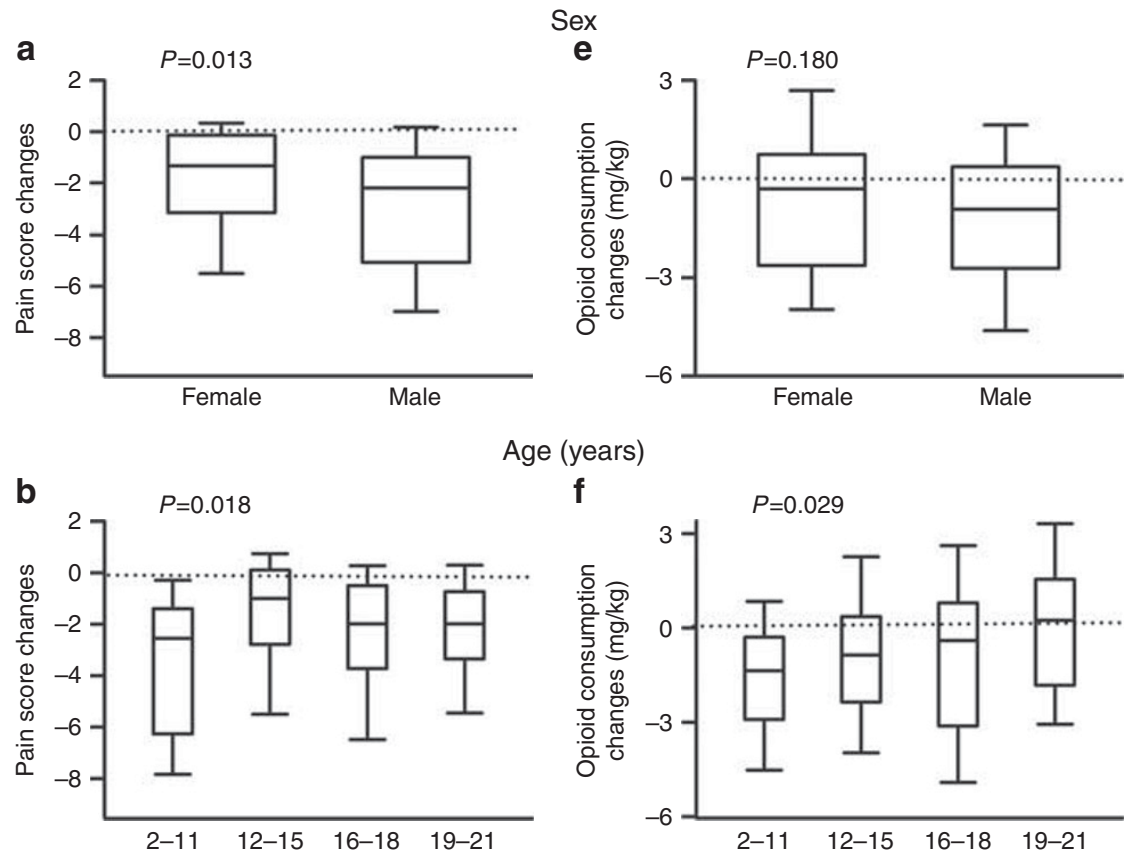

Age (years)
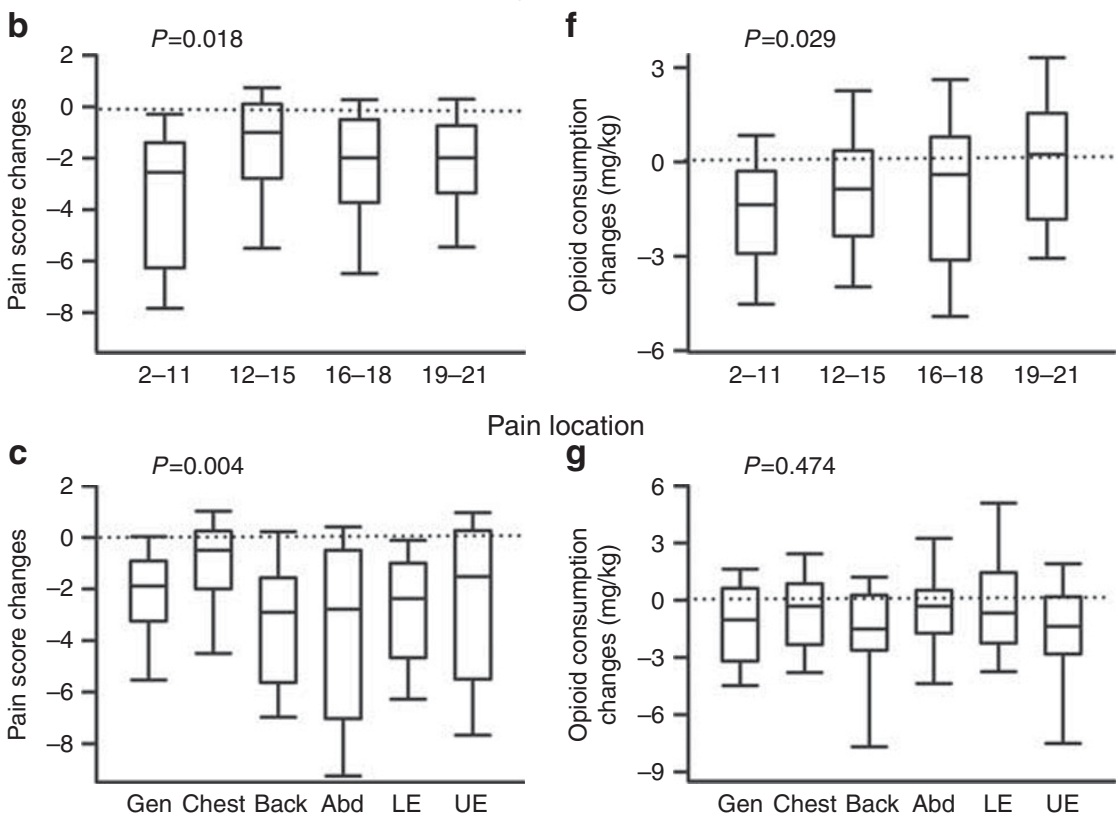

Pain location
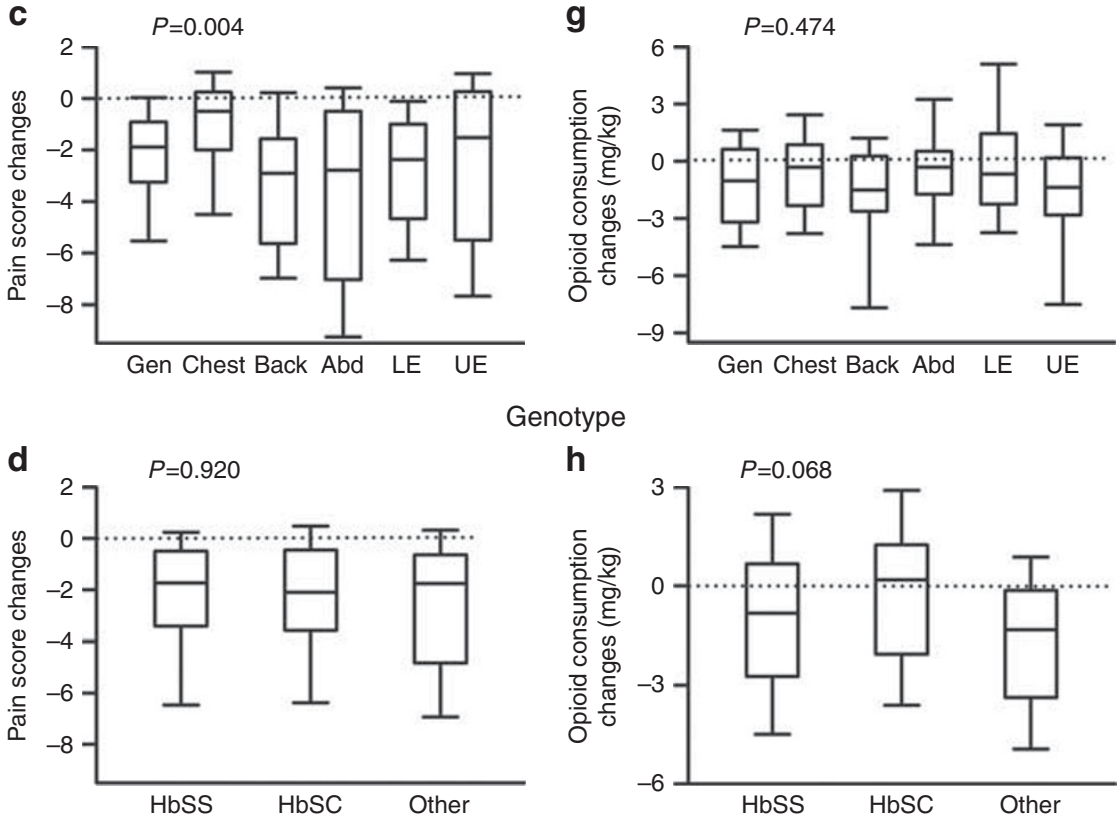

Figure 1. Changes in pain scores and opioid consumption. Differential effect of ketamine administration as an adjuvant to opioids on changes (from baseline to after ketamine) in pain scores (a-d) and opioid consumption (e-h) in SCD patients. Boxplots illustrate the median and interquartile range and the whiskers the 10th and 90th percentiles of the raw data. Gen indicates generalized pain defined as pain in 3 or more locations, Abd indicates abdomen, LE lower extremities, and UE upper extremities. The effect of ketamine infusions was sex-dependent, as it yielded greater reductions in pain scores in males compared with females $(\mathbf{a}, P=0.013)$. The effect of ketamine infusions on pain scores also varied according to age as in younger patients ketamine infusions yielded greater reductions in pain scores $(\mathbf{b}, P=0.018)$. With ketamine, greater reductions in pain scores were observed in patients with back and abdominal pain, whereas lesser reductions, in patients with chest pain (c, $P=0.004$, for overall difference). Ketamine effect on pain scores did not vary with genotype (d). The effect of ketamine on opioid consumption did not vary with sex (e, $P=0.180)$. In contrast, it varied with age as ketamine administered to patients of 2-11 years yielded greater and those administered to patients of 19-21 years yielded lesser decrease in opioid consumption (f, $P=0.029)$. Pain location had no impact on the effects of ketamine on opioid consumption $(\mathbf{g}, P=0.474)$. Ketamine infusions administered to patients with $\mathrm{HbSC}$ showed a trend toward lesser reductions in opioid consumption compared with other genotypes (h, $P=0.068)$. SCD, sickle cell disease. 


\section{Articles | Nobrega et al.}

infusion duration were independent predictors of changes in pain scores (Table 4). Male sex and longer infusions were predictors of greater reductions in pain scores compared with female sex and shorter infusions. In contrast, the presence of chest pain and ages between 12 and 15 years were predictors of lesser reductions compared with generalized pain and ages between 2 and 11 years (Table 4). There was no patient or infusion characteristic that independently predicted changes in opioid consumption.
We also examined possible predictors of clinically meaningful reductions in pain scores and opioid consumption. Sex, age, pain location, and genotype were independent predictors of clinically meaningful changes in pain scores. Males were significantly more likely to have a meaningful reduction in pain scores than females. Patients with ages between 12 and 18 years (compared with ages between 2 and 11), pain located in the chest (compared with generalized pain), and with genotypes $\mathrm{HbS} / \beta^{0}$ thalassemia, $\mathrm{HbS} / \beta^{+}$thalassemia, or $\mathrm{HbSS} / \alpha$ thalasse-
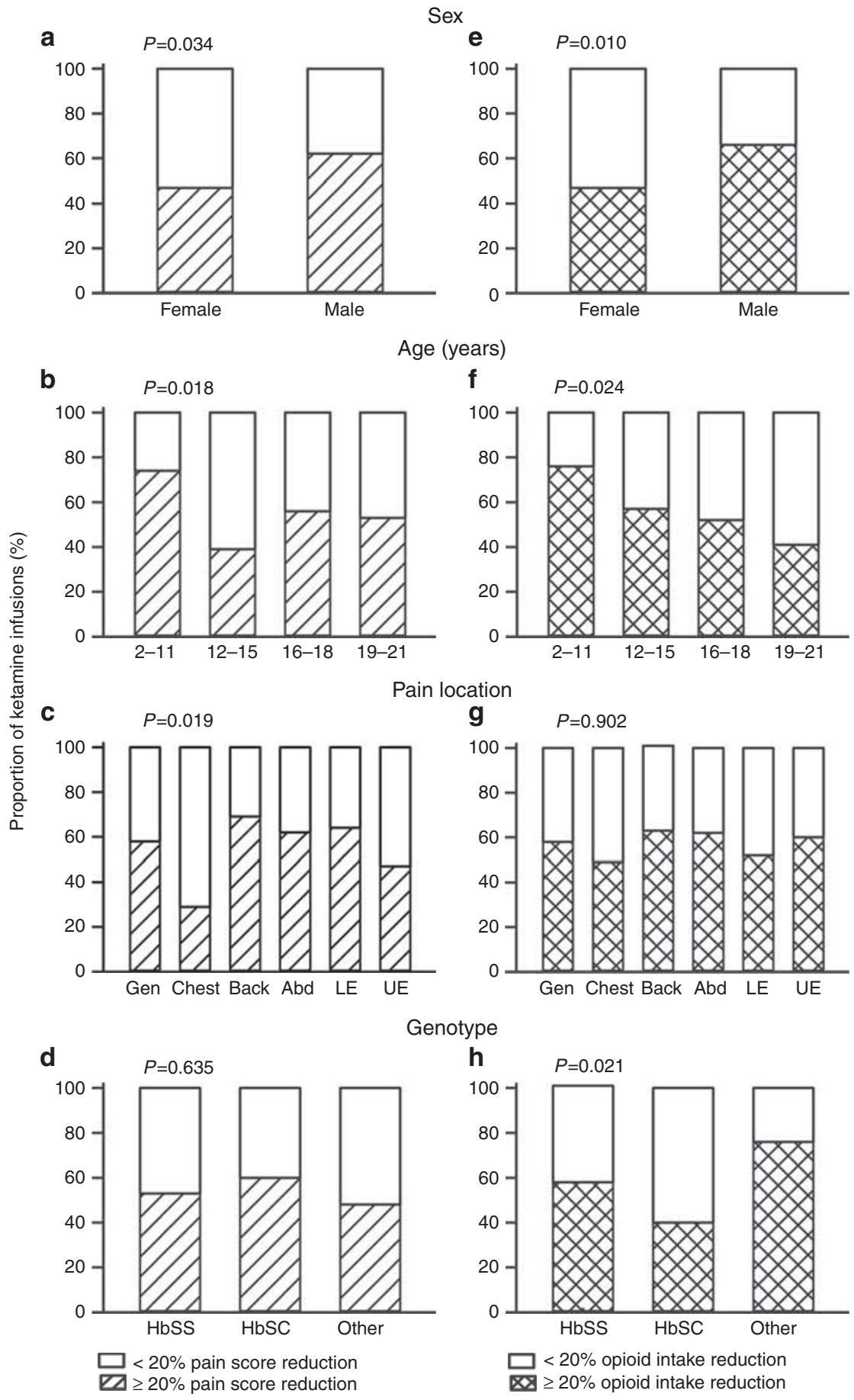
mia (compared with HbSS) were less likely to have meaningful reductions in pain scores (Table 5). There was also a trend toward increased odds of having a meaningful reduction in pain score in infusions administered to patients with $\mathrm{HbSC}$ genotype compared with HbSS (Table 5, $P=0.058$ ).

The only predictor of a meaningful reduction in opioid consumption was infusion duration. Patients receiving ketamine for 3 or 4 days were more likely to have meaningful reductions in opioid consumption compared with those receiving 2-day infusions (Table 5).

\section{DISCUSSION}

We found that in a large series of SCD patients admitted for VOE, infusions of subanesthetic doses of ketamine in combination with opioids were associated with an overall decrease in pain intensity and opioid consumption. Interestingly, multivariate analyses indicated that several patient characteristics including sex, age, pain location, and infusion duration were independent predictors of changes in pain scores. Specifically, male sex, younger age, and longer infusion durations were independent predictors of greater reductions in pain scores. Conversely, pain located in the chest was a predictor of lesser reduction in pain scores compared with pain that was generalized. It is noteworthy that, although

Table 4. Predictors of changes in pain scores from baseline to the day after ketamine discontinuation ${ }^{a}$

\begin{tabular}{|c|c|c|}
\hline Variable & $\begin{array}{c}\text { Coefficient ( } 95 \% \text { confidence } \\
\text { interval) }\end{array}$ & $P$ value \\
\hline \multicolumn{3}{|l|}{ Changes in pain scores } \\
\hline Sex (male vs. female) & $-1.36(-2.20,-0.52)$ & 0.002 \\
\hline $\begin{array}{l}\text { Age group (years; } 12-15 \text { vs. } \\
2-11 \text { ) }\end{array}$ & $1.80(0.62,2.98)$ & 0.003 \\
\hline $\begin{array}{l}\text { Pain location (chest vs. } \\
\text { generalized) }\end{array}$ & $1.26(0.27,2.25)$ & 0.013 \\
\hline $\begin{array}{l}\text { Infusion duration (days; } \geq 7 \\
\text { vs. } 2 \text { days) }\end{array}$ & $-1.73(-3.01,-0.45)$ & 0.008 \\
\hline \multicolumn{3}{|c|}{$\begin{array}{l}\text { a Multivariate mixed effects linear regression modeling was applied to the all- } \\
\text { admissions cohort and included patient number as a random effect in order to } \\
\text { account for the fact that individual patients contributed data from multiple hospital } \\
\text { admissions. Post hoc tests were then used to identify statistically significant differ- } \\
\text { ences within the subgroups of individual patient characteristics. }\end{array}$} \\
\hline
\end{tabular}

predictive of pain score reductions, no patient (age, sex, and pain location) or infusion characteristics (duration) were predictive of reductions in opioid consumption. Further, in this large series of SCD patients, we showed that the use of ketamine infusions in combination with opioids in regular patient care units was feasible and appeared to be safe. Thus, these findings generate the hypotheses that ketamine might have age- and sex-dependent effects in patients with SCD admitted with VOE-associated pain.

The finding that during ketamine and opioid infusions sex was an independent predictor of reductions in pain scores and

Table 5. Predictors of clinically meaningful reduction in pain scores and opioid consumption ${ }^{\mathrm{a}}$

\begin{tabular}{|c|c|c|}
\hline Variable & $\begin{array}{l}\text { Odds ratio ( } 95 \% \\
\text { confidence interval) }\end{array}$ & $P$ value \\
\hline \multicolumn{3}{|c|}{ Clinically meaningful reduction in pain scores } \\
\hline \multicolumn{3}{|l|}{ Sex } \\
\hline Male vs. female & $4.47(1.75,11.45)$ & 0.002 \\
\hline \multicolumn{3}{|l|}{ Age group (years) } \\
\hline $12-15$ vs. $2-11$ & $0.07(0.02,0.32)$ & 0.001 \\
\hline $16-18$ vs. $2-11$ & $0.20(0.05,0.80)$ & 0.023 \\
\hline \multicolumn{3}{|l|}{ Pain location } \\
\hline Chest vs. generalized & $0.19(0.06,0.55)$ & 0.002 \\
\hline \multicolumn{3}{|l|}{ Genotype } \\
\hline HbSC vs. HbSS & $2.42(0.97,6.03)$ & 0.058 \\
\hline Other vs. HbSS & $0.16(0.03,0.72)$ & 0.018 \\
\hline \multicolumn{3}{|c|}{ Clinically meaningful reduction in opioid consumption } \\
\hline \multicolumn{3}{|l|}{ Infusion duration (days) } \\
\hline 3 vs. 2 & $5.62(1.71,18.40)$ & 0.004 \\
\hline 4 vs. 2 & $3.94(1.16,13.40)$ & 0.028 \\
\hline \multicolumn{3}{|c|}{$\begin{array}{l}\text { aultivariate mixed effects logistic regression modeling was applied to the all- } \\
\text { admissions cohort and included patient number as a random effect in order to } \\
\text { account for the fact that individual patients contributed data from multiple hospital } \\
\text { admissions. Post hoc tests were then used to identify statistically significant differ- }- \text { ences within the subgroups of individual patient characteristics. Clinically mean- } \\
\text { ingful reduction was defined as a } 20 \% \text { or greater reduction in pain scores or in } \\
\text { opioid consumption (daily oral morphine-equivalent) from baseline to the day after } \\
\text { ketamine discontinuation (day after ketamine - first day of ketamine/first day } \times 100 \text { ). } \\
\mathrm{Hb} \text { represents hemoglobin, the other genotype group includes } \mathrm{HbS} / \beta^{\circ} \text { thalassema, } \\
\mathrm{HbS} / \beta^{+} \text {thalassemia, and HbSS/a thalassemia. }\end{array}$} \\
\hline
\end{tabular}

Figure 2. Proportion of ketamine infusions associated with a clinically meaningful reduction $(20 \%$ or greater reduction from baseline to the day after ketamine discontinuation) in pain scores (a-d) and opioid consumption (e-h). The stacked bars show the proportion of infusions associated with meaningful (hatched) and non-meaningful (white) reductions. A greater proportion of ketamine infusions administered to males was associated with meaningful reduction in pain scores compared with those administered to females $(\mathbf{a}, P=0.034)$. The highest proportion of infusions associated with meaningful reduction in pain scores was those administered to patients between 2 and 11 years of age and the lowest to those between 12 and 15 years $(\mathbf{b}, P=0.018)$. In addition, the highest proportion of infusions associated with a meaningful reduction in pain scores was those administered to patients with back, abdominal, and lower extremity pain and the lowest to patients with chest pain (c, $P=0.019)$. Genotype had no impact of the effect of ketamine in pain scores $(\mathbf{d}, P=0.635)$. Regarding opioid consumption, a significantly greater proportion of ketamine infusions administered to males was associated with a meaningful reduction in opioid consumption compared with those administered to females $(\mathbf{e}, P=0.010)$. The highest proportion of infusions associated with meaningful reduction in opioid consumption was those administered to patients between 2 and 11 years and the lowest to those between 19 and 21 years ( $f, P=0.024)$. Pain location had no impact on ketamine-associated changes on opioid consumption $(\mathbf{g})$. In addition, the lowest proportion of infusions associated with a meaningful reduction in opioid consumption was those administered to patients with $\mathrm{HbSC}$ and the greatest was those administered to patients with $\mathrm{HbS} / \beta^{0}$ thalassemia, $\mathrm{HbS} / \beta^{+}$thalassemia, or $\mathrm{HbSS} / \mathrm{a}$ thalassemia $(\mathbf{h}, P=0.021)$. 


\section{Articles | Nobrega et al.}

of clinically meaningful reductions in pain is intriguing and had not been previously reported. However, this finding is in keeping with animal and human studies showing that the effect of NMDA receptor antagonists indeed varies with sex and age. For example, in mice, non-competitive antagonists of NMDA receptors modulate morphine analgesia in males, but not in females (29). In addition, ketamine yields changes in brain tissue levels of the neurotransmitters glutamate and aspartate in a sex-dependent manner. Specifically, in male mice, ketamine administration yields lower glutamate levels in the hippocampus, whereas in females it yields higher aspartate levels in the prefrontal cortex (30). These sex-dependent changes in neurotransmitter levels are in turn associated with sex-dependent antidepressant effects (30). In fact, female mice are more sensitive to the antidepressant effects of ketamine early after exposure, but the antidepressant effect of ketamine lasts longer in male mice (30). In humans, there is also some evidence to suggest that males might have a greater response to the antidepressant effects of ketamine than females (31). Therefore, the present findings that ketamine appears to be more effective in reducing pain scores in males, and that male sex was an independent predictor of the effects of ketamine on pain intensity suggest that SCD male patients may be more responsive to NMDA receptor antagonism than female patients.

Age was also an independent predictor of the changes in pain scores with ketamine and opioid infusions. Compared with younger children, older SCD patients had less clinically meaningful reductions in opioid consumption and in pain scores. That ketamine can have age-dependent effects has been shown in animals $(32,33)$. Researchers have shown that anesthesia depth, measured by sleeping time, and the production of ketamine-active metabolites such as norketamine varies according to age in rodents $(32,33)$. It is possible that because of differences in pharmacokinetics of ketamine at the ages examined here, the effect of ketamine varied according to age. Alternatively, given the growing body of literature suggesting that patients with SCD can have changes in pain phenotype as they age, it is possible that the mechanisms underlying these changes in pain phenotype render their pain less responsive to NMDA receptor antagonism. Nevertheless, when designing clinical trials of ketamine in SCD patients, one needs to control for the effects of age and sex.

The determination of whether changes in pain scores or in opioid consumption are clinically meaningful is relevant during pain management as well as during the evaluation of analgesic efficacy. For adults with chronic pain, the Initiative on Methods, Measurement, and Pain Assessment in Clinical Trials consensus statement recommends that a $30 \%$ or greater change in pain intensity (measure by the NRS) be considered a moderate clinically significant change (34). However, no consensus recommendation has been established for children. In settings of acute pain, such as in the postoperative period, the minimal clinically meaningful difference is estimated to be a reduction of approximately one point on the NRS (from 0 to 10) (35). In children with chronic pain, one point or a $12.5 \%$ reduction on NRS-measured pain scores constitutes the minimal clinically meaningful difference (25). In SCD adolescents being treated for pain, others have shown that a mean change in pain score of $0.9(\mathrm{SE}=0.24), \sim 12.5 \%$ points measured on the NRS or of $0.97 \mathrm{~cm}$ on the visual analog scale is associated with the minimal clinically meaningful change and with the patient's perception of pain relief (26). For the purpose of this study, we arbitrarily defined clinically meaningful reduction in pain to be a $20 \%$ or greater reduction in pain scores. We showed that in this series the mean reduction in pain scores was of 2.87 points and noted that in $54 \%$ of ketamine infusions patients had a clinically meaningful pain reduction. Therefore, these findings suggest that in SCD patients ketamine in combination with opioid is associated with clinically significant reductions in pain intensity during admissions for VOEs.

Although in the cases examined here we did not identify any record of psychotomimetic effects that warranted treatment, ketamine is known to have psychoactive properties. In adults, ketamine is associated with increased psychotic symptoms both in healthy controls and subjects with history of schizophrenia $(36,37)$. Therefore, there is a theoretical concern that ketamine could exacerbate some psychiatric illnesses. Given the findings reported by others that SCD patients have a high incidence of associated mental health diagnoses and that patients with those diagnoses have a higher admission rate for VOEs (38), the theoretical concern that ketamine could exacerbate psychiatric symptoms certainly exists. Conversely, researchers have shown that depression is highly prevalent in SCD patients and that depressed patients have significantly higher pain burden than non-depressed subjects (39). Given the demonstrated antidepressant effect of ketamine, it is conceivable that some of the salutary effects of ketamine on pain intensity could be partially related to its antidepressant effects (40). Therefore, patient selection is certainly important when entertaining administration of ketamine to SCD patients. We also note that, although ketamine can have cardiovascular effects, we did not find any evidence that ketamine was associated with hemodynamic changes that warranted therapy with vasoactive agents. Taken together, these findings demonstrate the feasibility of administration of ketamine as an adjuvant to opioids to SCD patients admitted to inpatient care units with VOEs. In addition, although we are unable to draw definitive conclusions about the safety of ketamine as an adjuvant to opioids in SCD patients, in this series of SCD patients in the care setting described, ketamine was not associated with hemodynamic or neuropsychiatric effects that required therapy.

This study has limitations, which call for caution in the interpretation of its results. First, the study was conducted on data collected retrospectively and, although this is the largest reported series of ketamine administration to SCD patients, the examined population is small. In addition, the indication for ketamine administration was heterogeneous and included clinical impression of opioid tolerance, opioid hyperalgesia, or 
lack of response to opioid during admissions for VOEs. Another limitation is that we did not control for the presence of pre-existing chronic pain or history of previous admissions as the information was unavailable in many of the patients studied. Further, ketamine dose, duration of administration, and opioid titration were left to the discretion of those in charge of the patient's clinical care and, as a result, the treatment regimen was non-uniform. Most importantly, the fact that we did not have a control treatment arm precludes any conclusion about the efficacy of ketamine in SCD patients. Nevertheless, this study generates several questions, including the hypothesis that the effect of ketamine varies with sex, age, and pain location, all of which are worthy of testing in future studies as to determine which subset of SCD patients would benefit most from this therapeutic strategy. Further, this study generated data to inform the effect and sample size calculations for planning a randomized trial of ketamine in SCD patients. Those future studies will in turn lead to a deeper understanding of the role of NMDA antagonists for symptom management during VOEs in SCD patients.

\section{ACKNOWLEDGMENTS}

We thank the nursing staff and the members of the Division of Anesthesiology, Pain and Perioperative Medicine from the Children's National Health System for their outstanding care of our patients and their families.

\section{STATEMENT OF FINANCIAL SUPPORT}

This study was funded by a grant from the Sheik Zayed Institute for Pediatric Surgical Innovation. This manuscript was prepared while Z.M.N.Q. was employed at The Children's Research Institute, Children's National Health System.

\section{DISCLAIMER}

The opinions expressed in this article are the authors' own and do not necessarily reflect the view of the National Institutes of Health, the Department of Health and Human Services, or the United States government.

Disclosure: The authors declare no conflict of interest.

\section{REFERENCES}

1. Platt OS, Thorington BD, Brambilla DJ, et al. Pain in sickle cell disease. Rates and risk factors. N Engl J Med 1991;325:11-6.

2. Smith WR, Penberthy LT, Bovbjerg VE, et al. Daily assessment of pain in adults with sickle cell disease. Ann Intern Med 2008;148:94-101.

3. Ballas SK, Gupta K, Adams-Graves P. Sickle cell pain: a critical reappraisal. Blood 2012;120:3647-56.

4. Yawn BP, Buchanan GR, Afenyi-Annan AN, et al. Management of sickle cell disease: summary of the 2014 evidence-based report by expert panel members. JAMA 2014;312:1033-48.

5. Meier ER, Rampersad A. Pediatric sickle cell disease: past successes and future challenges. Pediatr Res 2017;81:249-58.

6. Zempsky WT, Loiselle KA, McKay K, et al. Retrospective evaluation of pain assessment and treatment for acute vasoocclusive episodes in children with sickle cell disease. Pediatr Blood Cancer 2008;51:265-8.

7. Wilkie DJ, Molokie R, Boyd-Seal D, et al. Patient-reported outcomes: descriptors of nociceptive and neuropathic pain and barriers to effective pain management in adult outpatients with sickle cell disease. J Natl Med Assoc 2010;102:18-27.

8. Brandow AM, Farley RA, Panepinto JA. Neuropathic pain in patients with sickle cell disease. Pediatr Blood Cancer 2014;61:512-7.

9. McNicol ED, Midbari A, Eisenberg E. Opioids for neuropathic pain. Cochrane Database Syst Rev 2013;8:CD006146.
10. Dampier C, Palermo TM, Darbari DS, Hassell K, Smith W, Zempsky W. AAPT diagnostic criteria for chronic sickle cell disease pain. J Pain 2017;18:490-8.

11. Tawfic QA. A review of the use of ketamine in pain management. J Opioid Manag 2013;9:379-88.

12. Sheehy KA, Muller EA, Lippold C, Nouraie M, Finkel JC, Quezado ZMN. Subanesthetic ketamine infusions for the treatment of children and adolescents with chronic pain: a longitudinal study. BMC Pediatrics 2015;15:198.

13. Michelet D, Hilly J, Skhiri A, et al. Opioid-sparing effect of ketamine in children: a meta-analysis and trial sequential analysis of published studies. Paediatr Drugs 2016;18:421-33.

14. Wang L, Johnston B, Kaushal A, Cheng D, Zhu F, Martin J. Ketamine added to morphine or hydromorphone patient-controlled analgesia for acute postoperative pain in adults: a systematic review and meta-analysis of randomized trials. Can J Anaesth 2016;63:311-25.

15. Latremoliere A, Woolf CJ. Central sensitization: a generator of pain hypersensitivity by central neural plasticity. J Pain 2009;10:895-926.

16. Angst MS, Koppert W, Pahl I, Clark DJ, Schmelz M. Short-term infusion of the mu-opioid agonist remifentanil in humans causes hyperalgesia during withdrawal. Pain 2003;106:49-57.

17. Nielsen RV, Fomsgaard JS, Siegel H, et al. Intraoperative ketamine reduces immediate postoperative opioid consumption after spinal fusion surgery in chronic pain patients with opioid dependency: a randomized, blinded trial. Pain 2017;158:463-70.

18. Neri CM, Pestieau SR, Young H, Elmi A, Finkel JC, Darbari DS. Low-dose ketamine for children and adolescents with acute sickle cell disease related pain: a single center experience. J Anesthe Clin Res 2014;5.

19. Neri CM, Pestieau SR, Darbari DS. Low-dose ketamine as a potential adjuvant therapy for painful vaso-occlusive crises in sickle cell disease. Paediatr Anaesth 2013;23:684-9.

20. Zempsky WT, Loiselle KA, Corsi JM, Hagstrom JN. Use of low-dose ketamine infusion for pediatric patients with sickle cell disease-related pain: a case series. Clin J Pain 2010;26:163-7.

21. Tawfic QA, Faris AS, Kausalya R. The role of a low-dose ketaminemidazolam regimen in the management of severe painful crisis in patients with sickle cell disease. J Pain Symptom Manage 2014;47:334-40.

22. Sheehy KA, Finkel JC, Darbari DS, Guerrera MF, Quezado ZM. Dexmedetomidine as an adjuvant to analgesic strategy during vaso-occlusive episodes in adolescents with sickle-cell disease. Pain Pract 2015;15:E90-7.

23. Fein DM, Avner JR, Scharbach K, Manwani D, Khine H. Intranasal fentanyl for initial treatment of vaso-occlusive crisis in sickle cell disease. Pediatr Blood Cancer 2016;64. (e-pub ahead of print).

24. Mercadante S, Ferrera P, Villari P, Casuccio A, Intravaia G, Mangione S. Frequency, indications, outcomes, and predictive factors of opioid switching in an acute palliative care unit. J Pain Symptom Manage 2009;37:632-41.

25. Hirschfeld G, Wager J, Schmidt P, Zernikow B. Minimally clinically significant differences for adolescents with chronic pain-variability of ROC-based cut points. J Pain 2014;15:32-9.

26. Myrvik MP, Brandow AM, Drendel AL, Yan K, Hoffmann RG, Panepinto JA. Clinically meaningful measurement of pain in children with sickle cell disease. Pediatr Blood Cancer 2013;60:1689-95.

27. Sheehy KA, Lippold C, Rice AL, Nobrega R, Finkel JC, Quezado ZM. Subanesthetic ketamine for pain management in hospitalized children, adolescents, and young adults: a single-center cohort study. J Pain Res 2017;10:787-95.

28. Finkel JC, Pestieau SR, Quezado ZM. Ketamine as an adjuvant for treatment of cancer pain in children and adolescents. J Pain 2007;8:515-21.

29. Nemmani KV, Grisel JE, Stowe JR, Smith-Carliss R, Mogil JS. Modulation of morphine analgesia by site-specific N-methyl-D-aspartate receptor antagonists: dependence on sex, site of antagonism, morphine dose, and time. Pain 2004;109:274-83.

30. Franceschelli A, Sens J, Herchick S, Thelen C, Pitychoutis PM. Sex differences in the rapid and the sustained antidepressant-like effects of ketamine in stress-naive and "depressed" mice exposed to chronic mild stress. Neuroscience 2015;290:49-60. 


\section{Articles | Nobrega et al.}

31. Coyle CM, Laws KR. The use of ketamine as an antidepressant: a systematic review and meta-analysis. Hum Psychopharmacol 2015;30: 152-63.

32. Waterman AE, Livingston A. Effects of age and sex on ketamine anaesthesia in the rat. Br J Anaesth 1978;50:885-9.

33. Giroux MC, Santamaria R, Helie P, Burns P, Beaudry F, Vachon P. Physiological, pharmacokinetic and liver metabolism comparisons between 3-, 6-, 12- and 18-month-old male Sprague Dawley rats under ketamine-xylazine anesthesia. Exp Anim 2016;65:63-75.

34. Dworkin RH, Turk DC, Wyrwich KW, et al. Interpreting the clinical importance of treatment outcomes in chronic pain clinical trials: IMMPACT recommendations. J Pain 2008;9:105-21.

35. Voepel-Lewis T, Burke CN, Jeffreys N, Malviya S, Tait AR. Do 0-10 numeric rating scores translate into clinically meaningful pain measures for children? Anesth Analg 2011;112:415-21.
36. Xu K, Krystal JH, Ning Y, et al. Preliminary analysis of positive and negative syndrome scale in ketamine-associated psychosis in comparison with schizophrenia. J Psychiatr Res 2015;61:64-72.

37. Lahti AC, Weiler MA, Tamara Michaelidis BA, Parwani A, Tamminga CA. Effects of ketamine in normal and schizophrenic volunteers. Neuropsychopharmacology 2001;25:455-67.

38. Myrvik MP, Burks LM, Hoffman RG, Dasgupta M, Panepinto JA. Mental health disorders influence admission rates for pain in children with sickle cell disease. Pediatr Blood Cancer 2013;60:1211-4.

39. Levenson JL, McClish DK, Dahman BA, et al. Depression and anxiety in adults with sickle cell disease: the PiSCES project. Psychosom Med 2008;70:192-6.

40. Diazgranados N, Ibrahim L, Brutsche NE, et al. A randomized add-on trial of an N-methyl-D-aspartate antagonist in treatment-resistant bipolar depression. Arch Gen Psychiatry 2010;67:793-802. 